\title{
Lesson of the month 2: A choroid plexus papilloma manifesting as anorexia nervosa in an adult
}

\author{
Authors: Prateush Singh, ${ }^{\mathrm{A}}$ Asim Khan, ${ }^{\mathrm{B}}$ Georgia Scott, ${ }^{\mathrm{C}}$ Esha Singh ${ }^{\mathrm{D}}$ and Manuel Jasper ${ }^{\mathrm{E}}$
}

\begin{abstract}
A Caucasian female previously diagnosed with anorexia nervosa was referred by psychiatric services to the general medical team. She presented with dehydration, vomiting, weakness, a body mass index of $13 \mathrm{~kg} / \mathrm{m}^{2}$ and was treated with intravenous and enteral supplementation. During admission her vomiting worsened and she developed visual hallucinations and confabulation. Neurological examination demonstrated cerebellar signs and bilateral papilloedema on fundoscopy. Subsequent magnetic resonance imaging of the brain revealed a large fourth ventricular tumour causing obstructive hydrocephalus. The tumour was excised and histologically confirmed to be a choroid plexus papilloma. Postoperatively her neurological symptoms and negative feelings towards eating resolved.
\end{abstract}

KEYWORDS: Anorexia nervosa, brain tumour, choroid plexus papilloma

\section{Introduction}

Anorexia nervosa is a debilitating psychiatric condition with significant medical sequelae. Most cases are associated with predisposing, precipitating and perpetuating psychosocial factors. We were able to establish an organic cause (a brain tumour) for our patient's signs and symptoms.

Rare organic causes, such as brain tumours, can present with clinical features indicative of anorexia nervosa; these are more likely to be hypothalamic or frontal lobe tumours. Our patient had a fourth ventricular tumour, a choroid plexus papilloma, which is exceptionally rare in the adult population. Nevertheless, one should have a low investigative threshold for excluding appropriate organic causes prior to mislabelling biological symptoms as psychiatric.

\section{Case history}

A 38-year-old Caucasian female diagnosed with anorexia nervosa and anxiety 18 months previously was referred to the

Authors: ${ }^{\mathrm{A}} \mathrm{FY}$ 2, Basildon University Hospital, Basildon, UK; ${ }^{\mathrm{B}} \mathrm{CT} 2$, Basildon University Hospital, Basildon, UK; ${ }^{C}$ FY1, Basildon University Hospital, Basildon, UK; ${ }^{D}$ medical student, Basildon Hospital, Basildon, UK; ${ }^{E}$ consultant gastroenterologist, Basildon University Hospital, Basildon, UK medical team with dehydration, fatigue and further weight loss. She had had multiple similar admissions in the recent past, was treated symptomatically then discharged to the community eating disorders team. Collateral history revealed that her psychiatric problems coincided with unemployment and relationship difficulties. She was treated with citalopram and quetiapine by the psychiatric team in an attempt to improve her anxiety and nutritional intake.

On examination she weighed $30 \mathrm{~kg}$ with a body mass index (BMI) of $13 \mathrm{~kg} / \mathrm{m}^{2}$. Examination of her major organ systems was unremarkable although cerebellar examination was not initially performed. Her abbreviated mental test score was 10/10. Most blood tests - including blood count, renal, liver and thyroid function, haematinics, glucose and calcium - were unremarkable. Serum potassium, phosphate and magnesium levels were low at 3.2, 0.70, and $0.72 \mathrm{mmol} / \mathrm{L}$, respectively, and serum cortisol was raised $(760 \mathrm{mmol} / \mathrm{L})$, consistent with malnutrition and a high risk of refeeding syndrome. Oesophago-gastro-duodenoscopy and abdominal ultrasound were performed to investigate her vomiting and revealed no abnormalities.

Her dehydration and malnourishment were treated with enteral and parenteral replacement. During admission she refused meals and deliberated self-discharge but she was deemed to lack capacity and a deprivation of liberty safeguard was instigated to prevent her leaving the ward. She agreed to nasogastric insertion and her weight increased to $35 \mathrm{~kg}$ over 2 weeks. Her blood tests remained normal thereafter.

Despite nutritional support she developed transient visual hallucinations and confabulation. Neurological examination revealed left-sided nystagmus, bilateral dysmetria, pastpointing, hypotonia, dysdiadochokinesia and intention tremor. Fundoscopy demonstrated bilateral chronic papilloedema. Capillary blood glucose, blood gas, electrocardiogram, urinalysis and chest X-ray were unremarkable. Urgent magnetic resonance imaging (MRI) of the brain illustrated an intensely enhancing tumour in the fourth ventricle measuring $49 \times 24 \mathrm{~mm}$ (Fig 1) and causing obstructive hydrocephalus. The lateral ventricles showed significant dilatation with periventricular trans-ependymal oedema (Fig 2) and effacement of the sulci but no evidence of other lesions throughout the remaining brain parenchyma. She was immediately started on intravenous dexamethasone to reduce intracranial pressure. 


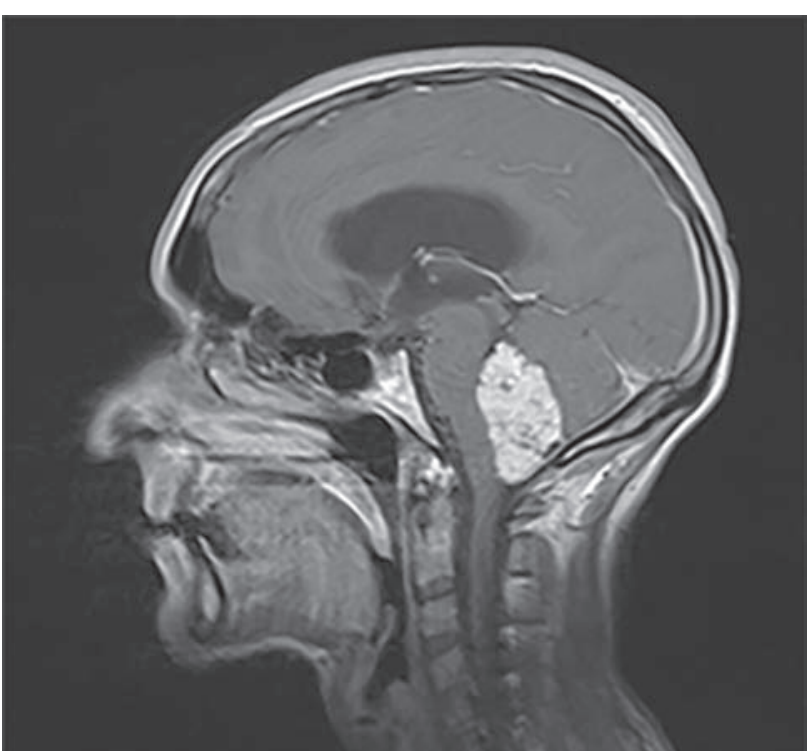

Fig 1. Sagittal T1-weighted magnetic resonance image demonstrating the fourth ventricular brain tumour.

A computerised tomography (CT)-chest-abdomen-pelvis scan was done on neurosurgical recommendation and excluded a primary tumour elsewhere. Although not sensitive to exclude small primary tumours, skin malignancies like melanomas or cancers in the face and neck, it is a valuable imaging modality for excluding large primary tumours in a large proportion of the body. An MRI spine was then done to discount spinal cord seeding. With the radiological characteristics of the tumour, the clinical impression, and the negative CT and spinal MRI, it was assumed that the brain tumour was primary with

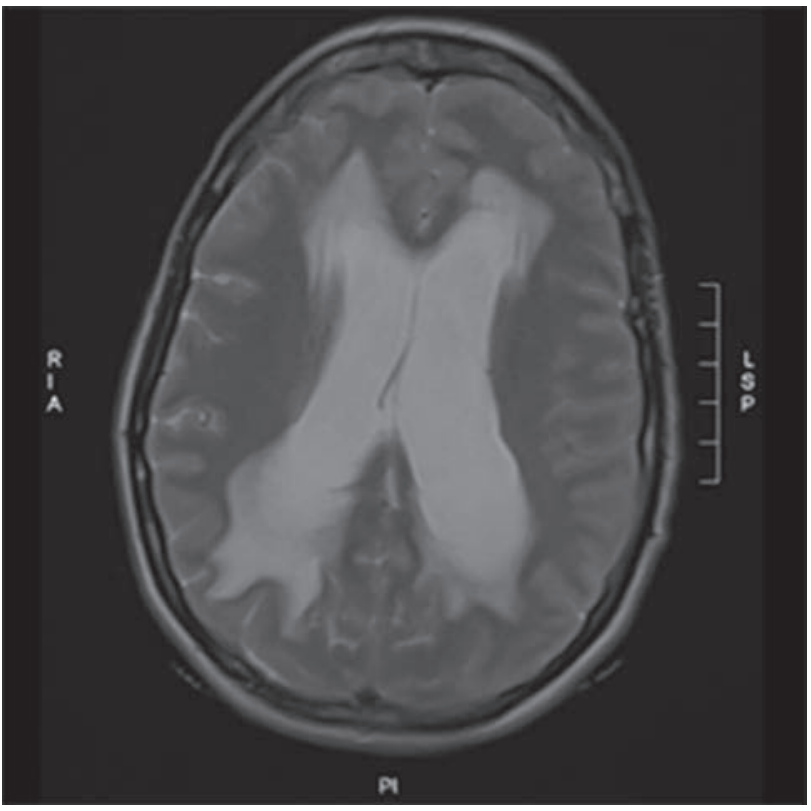

Fig 2. Axial T2-weighted magnetic resonance image demonstrating hydrocephalus. differentials including ependymoma, choroid plexus papilloma or medulloblastoma. The tumour was completely resected and histologically confirmed to be a choroid plexus papilloma excised with clear margins. A follow-up MRI revealed the tumour to have been completely resected with no recurrence.

Postoperatively, all of her neurological symptoms resolved and her feelings and behaviour towards eating improved dramatically. Four months later, her weight exceeded $50 \mathrm{~kg}$ and her BMI was $19 \mathrm{~kg} / \mathrm{m}^{2}$. This is sufficient evidence to suggest that the tumour was causative for her anorexia.

\section{Discussion}

Choroid plexus papillomas are rare, slow-growing, benign neoplasms comprising less than $1 \%$ of all brain tumours. They typically affect the lateral ventricles in children but the fourth ventricle and cerebellopontine angle in adults. Over $80 \%$ occur in children under 1 year old. Clinical features include headaches, vomiting, visual disturbances, papilloedema, seizures, drowsiness and focal neurology. Rarely, they can present with psychotic or behavioural disturbances. ${ }^{1}$

Our patient presented with vomiting, weight loss and clinical features mimicking anorexia nervosa. A comprehensive neurological examination was not performed on admission, which may have elicited cerebellar signs earlier. A thorough clinical assessment should be performed when features suggesting organ pathology are present - the vomiting should have warranted a complete neurological examination.

Although her social circumstances may have contributed to her mislabelled initial diagnosis, her symptoms actually predated these events and should have been investigated first. Organic causes should be excluded prior to labelling symptoms as psychiatric, as per the World Health Organization International Classification of Diseases (ICD-10). As our patient had an established diagnosis of anorexia nervosa, the assumption that her vomiting was secondary to this alone was falsely presumed.

The medical treatment of complications related to anorexia nervosa is a complex ethical area and the Mental Capacity Act and Mental Health Act should be instigated appropriately. A multidisciplinary approach involving the patient, family, dietician, nurses and psychiatrists is imperative.

Hypothalamic, fronto-temporal tumours and craniopharyngiomas have been known to masquerade as eating disorders. ${ }^{2-4}$ Rarely, brain tumours affecting the fourth ventricle have also been implicated in the development of eating disorders. ${ }^{2}$ Removal of the tumours in all these cases resulted in a reversal of the eating disorder.The pathophysiological connection between brain tumours and reduced appetite to the point of anorexia nervosa is not well understood. Further research in to this area may help to understand the association.

\section{Conflicts of interest}

The authors have no conflicts of interest to declare.

\section{Author contributions}

All authors have contributed equally in the management of the patient in this case and in the subsequent literature review, gathering consent and images and writing the manuscript. They meet all the authorship criteria of the ICMJE. 


\section{Consent to publish}

We have written informed consent from the patient to write this case and all images associated with. This is available to view by the editors at their discretion.

\section{Acknowledgements}

This case was presented as an oral presentation at the European Society of Surgical Research conference, Prague, 2016.

\section{References}

1 Gozali AE, Britt B, Shane L et al. Choroid plexus tumors; management, outcome, and association with the Li-Fraumeni syndrome: The Children's Hospital Los Angeles (CHLA) experience, 1991-2010. Pediatr Blood Cancer 2012;58:905-9.
2 Wright K, Smith MS, Mitchell J. Organic diseases mimicking atypical eating disorders. Clin Pediatr 1990;29:325-8.

3 De Vile CJ, Sufraz R, Lask BD, Stanhope R. Occult intracranial tumours masquerading as early onset anorexia nervosa. BMJ 1995;311:1359-60.

4 Trummer M, Eustacchio S, Unger F, Tillich M, Flaschka G. Right hemispheric frontal lesions as a cause for anorexia nervosa report of three cases. Acta Neurochir 2002;144:797-801.

Address for correspondence: Dr Prateush Singh, Department of Gastroenterology, Basildon University Hospital,

Nethermayne, Basildon SS16 5NL, UK.

Email: singh.prateush@gmail.com 